Número especial “Convenção internacional sobre meio ambiente e desenvolvimento para integração e cooperação para a sustentabilidade" - Havana/Cuba.

Vol. 25, n. 1, 2020.

Artigo recebido em: 03/02/2020

Artigo aprovado em: 04/03/2020

\title{
CONCEPCIÓN COMUNITARIA DE LA EDUCACIÓN AMBIENTAL PARA EL ENFRENTAMIENTO Y ADAPTACIÓN AL CAMBIO CLIMÁTICO
}

\author{
CONCEPÇÃO COMUNITÁRIA DA EDUCAÇÃO AMBIENTAL PARA O \\ ENFRENTAMENTO E ADAPTAÇÃO À MUDANÇA CLIMÁTICA
}

\section{COMMUNITY CONCEPTION OF ENVIRONMENTAL EDUCATION FOR FACING AND ADAPTATION TO CLIMATE CHANGE}

\author{
Georgina Castro Acevedo'; \\ José Cebey ${ }^{2}$
}

\begin{abstract}
RESUMO
À mudança do clima é um dos dois fenômenos que mais preocupam a sociedade. O objetivo deste artigo é discutir o conceito de Educação Ambiental Comunitária não escolarizada, com base em uma posição teórico-metodológica dos autores que não ocorreu em 2016. Ou o trabalho de conceituação responde a dois pesquisadores. Para homenagear o fortalecimento do protagonismo dos sujeitos a partir de uma proposta de educação para o desenvolvimento, o viável não enfrentou e se adaptou às mudanças climáticas, além de assumir um papel importante na consolidação de uma educação inclusiva contida nos Objetivos de 2030. Sua aplicação tributária para gerar Comportamentos e atitudes responsáveis, bem como a vontade de assumir compromissos diante do confronto e adaptação às mudanças climáticas. À Concepção conta com técnicas e métodos de grupo para garantir participação e destaque aos sujeitos.
\end{abstract}

Palavras-chave: educação ambiental comunitária, mudança do clima.

\begin{abstract}
Climate change is one of the two phenomena that most concern society. The aim of this paper is to discuss the concept of non-schooled Community Environmental Education, based on a theoreticalmethodological position of the authors that did not occur in 2016. Or the conceptualization work answers two researchers. To honor the strengthening of the protagonism of the subjects from a development education proposal, the viable did not face and adapt to climate change, besides assuming an important role in the consolidation of an inclusive education contained in the 2030 Goals. To generate responsible behaviors and attitudes, as well as the willingness to make commitments in the face of confrontation and adaptation to climate change. The Conception has techniques and group methods to ensure participation and prominence to the subjects.
\end{abstract}

Keywords: community, environmental, education, climate change.

1Dra C de La Educación. Profesora Titular Del Centro de Estúdios Comunitarios de La Universidad Central "Marta Abreu" de Las Villas. Cuba, coordinadora de La Estratégia Ambiental en La Facultad de Ciencias Sociales, miembro del Instituto Virtual de Medio Ambiente de esa Universidad, del grupo de trabajo de cambio climático del CITMA VC; Del grupo de trabajo para estúdios de dinámicas poblacionales en la província de VC. E-mail geocast@uclv.edu.cu

2Máster en Didáctica de la Geografía. Profesor auxiliar del departamento de Estudios Socioculturales y Socilogia de La Universidad Central "Marta Abreu" de Las Villas. Cuba, miembro del Instituto Virtual de Medio Ambiente de esa Universidad E-mail joseantonio@uclv.edu.cu 


\section{INTRODUCCIÓN}

En el medio ambiente interactúan un conjunto de componentes donde el hombre es la especie más preciada. La relación entre los componentes naturales, socioeconómicos y socioculturales no siempre ha sido sostenible y está mediada, em ocasiones, por patrones de consumo que permanecen en el imaginario social de los sujetos que interactúan em cada espacio geográfico.

El espacio geográfico tiene particuparidades diferentes em cada contexto. Según (Santos,1992: 88 p) el espacio está formado por los objetos naturales, artificiales y por la sociedad. Según esse autor la esencia del espacio es social, se infiere por tanto que el comportamiento del médio ambiente em cada contexto está mediado por el manejo que la socidad hace de él y por el ordenamiento del território.

El desconocimieno del carácter social del espacio y del enfoque totalizador del médio ambiente condujo a la existência de los problemas ambientales que hoy enfrenta la humanidad. Reconocer a la sociedad insertada em la problemática ambiental y no como telón de fondo es um reto de la Educación Ambiental contemporánea (Castro, 2016: p 21).

Em esse sentido, destaca la autora, es necesario, repensar en el papel que cada ciencia puede aportar al análisis interdisciplinario de la problemática ambiental. Esa transformación del pensamiento conduce a la transformación social de los sujetos y los orienta a comprender el enfoque totalizador del Medio Ambiente (MA).

Para llegar a tal aspiración la ciencia debe buscar formas novedosas que tributen al protagonismo y la responsabilidad de cada sujeto individual y colectivo, al respeto y ética mostrados em cada acción que se realiza en el medio ambiente, de forma que a futuro, se minimicen los problemas ambientales y se alcance un uso sostenible del MA.

Para lograr tal propósito se puede recurrir a la Educación Ambiental (EA) la que como proceso educativo tiene un papel protagónico em los modos de actuación de la sociedad. Tal proceso debe proyectarse como dimensión (Roque, 2003: p15) Según esa autora, de esa forma, se transversalizan todos los procesos. 
Como contribución a esa idea (Castro, 2016: p 23) destaca la necesidad de indagar em lãs diferentes formas no escolarizadas como vía para grantizar la inclusión de toda la sociedad tal y como se aspira desde lãs Metas (2030). Desde ese proceso esencialmente educativo deben emerjer conocimientos, habilidades, valores, comportamientos, tipificados por el protagonismo de los sujetos.Según los autores del presente artículo, esa idea rectora deberá transversalizar los proyectos de desarrollo de cada contexto.

Desde la década del 90 del pasado siglo y como consecuencia de los logros de la Cumbre de Río 1992, la Educación Ambiental cubana desarrolló estrategias, metodologías y proyectos que tributaron al desarrollo de la cultura ambiental de la sociedad. Hoy exhibe entre sus logros el de contar con resultados satisfactorios em la proyección de La EA como dimensión. Esos logros van desde su inclusión em los Planes de Estudio y Programas del Sistema Nacional de Educación, hasta acciones comunitárias para la concientización de toda la población.

En Cuba los estudios del medio ambiente no se realizan desde uma Carrera, disciplina o asignatura sino que es um eje transversal que se incluye em los procesos educativos. La escuela como centro cultural más importantes del asentamiento es la protagonista de organizar las acciones de Educación Ambiental (EA) por La Via Formal y No Formal.

Tomando como antecedentes las valiosas experiencias desarrolladas en ese campo por investigadores no solo en Cuba, sino em otras nacionalidades como España, Brasil, México entre otras, los autores del presente artículo defienden una concepción comunitaria de La EA desde formas no escolarizadas que potencia la particpación, cooperación y conciencia critica del sujeto que se convierte en protagonista de nuevos proyectos (Castro, 2016: p 29).

La concepción de Educación Ambiental Comunitária no escolarizada (EAC) sin lugar a dudas enriquece la EA tradicional dirigida a resolver los problemas ambientales del espacio. Tiene como valor primero que se complementa con la forma tradicional, y segundo que reconoce a la sociedad como parte del médio ambiente en ese espacio, para lo cual otorga protagonismo a los sujetos.

Desde el 2010 hasta la fecha los autores han aplicado esa concepción em proyectos de investigación, y tesis de culminación de estudios de la Carrera de: 
Estudios Socioculturales en La Universidad Central "Marta Abreu" de Las Villas, Lo resultados avalan su valor en escenarios diversos de la provincia Villa Clara, Cuba como son los municípios de:(Quemado, Caibarien, Santa Clara; Manicaragua) entre otros.

La concepción teórico- metodológica asumida por los autores facilita que la sociedad esté contenida en el análisis de la problemática del medio ambiente y no como telón de fondo. Es decir; pasar de excluirla de su responsabilidad con la sostenibilidad de la vida en el planeta a ser parte de la problemática ambiental y participar de lãs acciones em pos de la sostenibilidad.

Una de las primeras propuestas en relación con la sostenibilidad se presentó en el Informe de Brutland (1987). Esa propuesta centra al ser humano y la satisfacción de sus necesidades en el nuevo estilo de desarrollo y aunque aspira a superar los antagonismos entre desarrollo y medio ambiente, es ambigua en el análisis de los costos del desarrollo sostenible y tampoco define qué estrategias se asumirán para eliminar la pobreza.

La postura del Desarrollo Sostenible (DS) planteado desde la última década del pasado siglo ha generado numerosas críticas como: esa concepción de desarrollo no identifica la existencia de sujetos que transformen la realidad, no ha tenido en cuenta que el planeta, las regiones y los territorios no viven en condiciones de homogenización espacial y tampoco que están sometidos a los principios de la globalización neoliberal.

A criterio de los autores la concepción del (DS) por su ambiguedad genera dos contradicciones: la primera está dada porque como concepción aspira a que las generaciones actuales dirijan las necesidades de generaciones futuras sin conocer las características de ese contexto. La segunda limita la participación de las generaciones futuras ya que asumen las necesidad prefijadas por generaciones anteriores lo que no les permite asumir sus propias propuestas en un contexto que será diferente, con contradicciones, problemas y significados diferentes.

Desde ese presupuesto en este trabajo el término desarrollo sostenible significa comprender el medio ambiente desde una visión integradora, donde el desarrollo está mediado por la concepción comunitaria, que genera participación y 
protagonismo de los sujetos en pos de la sostenibilidad ajustado a lãs perticularidades de cada contexto.

\section{DESARROLLO}

La propuesta teórica metodológica de Educación Ambiental Comunitaria no escolarizada que se defiende aqui, aspira desde su génesis a desarrollar la conciencia crítica de los sujetos, su participación y cooperación en torno a un proyecto común. Por tal fundamento se convierte en una importante herramienta para la proyección de acciones que tributen al desarrollo sostenible y a la proyección de las acciones para el cumplimiento de los objetivos del desarrollo Sostenible (2016).

Este artículo tiene como objetivo: argumentar teórica y metodológicamente la concepción de la Educación Ambiental Comunitaria no escolarizada que se asume para contribuir a desarrollar la cultura ambiental.de la sociedad. Se proyecta al enfrentamiento y adaptación al cambio climático, por ser uno de los problemas que afecta al planeta y al archipiélago cubano por su condicion insular. Desde esa concepción (Castro, 2016) destaca que:

- Se potencia el análisis e interpretación de los fenômenos

- Guía la investigación em pos de una educación ambiental comunitaria a partir de la práctica social como base teórica metodológica.

- Potencia la formacion conocimientos, habilidades, actitudes, comportamientos y valores desde formas no escolarizadas, lo que resulta novedoso para el desarrollo de investigaciones en relación con la adaptación al Cambio Climático Tarea Vida para Cuba.

Prevalece el análisis cualitativo, se enmarca en el referente teóricometodológico de la perspectiva del autodesarrollo comunitario propuesto por el Centro de Estudios Comunitarios de la Universidad Central "Marta Abreu" de Las Villas (2004), que valoriza el papel del proyecto colectivo y de la participación de los sujetos para la transformación de la realidad.tiene su génesis en la Investigación-Acción-Participación -Transformación

Aunque em Cuba existen experiencias exitosas en la educación ambiental comunitaria desde formas tradicionales, esta propuesta vigoriza el papel de la 
sociedad en el enfrentamiento aL cambio climático y es una contribución teórica metodológica al Proyecto de Resiliencia Ambiental Comunitaria para El desarrollo local (2019-2021), que ejecuta el Centro de Estudios Comunitarios, de la Universidad Central Marta Abreu de Las Villas en Cuba.

\section{LA REALIDAD DEL CAMBIO CLIMÁTICO, ENFRENTAMIENTO $Y$ TRANSFORMACIÓN: PROYECCIÓN EN CUBA.}

La preocupación por el cambio climático es una demanda de la sociedad actual, y lo es porque preocupa lo que pueda ocurrir a nosotros, a la familia, a los descendientes, por los efectos que provoca en todas las esferas de la vida.

Los investigadores de ese tema ayudan en gran medida, a satisfacer esa preocupación; pero en especial a la sociedad, no a los sujetos individuales, porque han sido los sujetos colectivos los que han hecho posible profundizar en los aspectos relacionados con el Cambio Climático

La idea del Cambio Climático (CC) surge en los años 70 del pasado siglo, el tema logró importar a la sociedad con una intensidad variable. Una sistematización teórica realizada al respecto indica que hasta mediados del 90 hubo un período de ascenso como objeto de atención, y luego una fase de decadencia.

Entre el 2003 y el 2006 se observa un incremento en la preocupación por el tema lo que se corrobora en encuestas aplicadas en diferentes países. En Europa el incremento de esa preocupación era inferior a los países sudamericanos, conforme Baigorri (2012). Aunque el CC es un problema serio para la sociedad, desde donde se han emitidos argumentos científicos en relación con el tema, existe otro grupo que no cree en este fenómeno alegando que en otras épocas geológicas del planeta han existido procesos similares de origen natural.

Desde ese presupuesto compete a la ciencia profundizar en el asunto, para introducir procesos educativos emancipatorios dirigidos a modificar, conocimientos, actitudes, valores dirigidos a la adaptación y enfrentamiento al CC. Cada vez es innegable que el futuro de la sociedad sea mediante adaptación, resiliencia, o enfrentamiento exige de profundas transformaciones.

Es la resiliencia ¿adaptación o enfrentamiento? A criterio de los autores de este articulo existe un nivel superior al que se aspira en relación con la resiliencia 
ambiental, ella está dada por la transformación de la sociedad. En ese sentido la cualidad comunitaria aporta un valor agregado que se da en la relaciones de los sujetos que comparten un proyecto común para enfrentarse a los efectos del CC:

Ese fenómeno constituye uno de los principales desafíos a los que se ha enfrentado la civilización moderna, es en sí mismo interés de las Ciencias Naturales y Sociales. De las segundas encontramos estudios de: (Jordán. 2009, Duarte,2006, Rodríguez, 2008,) citados por (Baigorri, 2012: p 67). En la sistematización de sus estudios se aprecia que esos investigadores se centran en la percepción social del CC y en la conducta de la población. Se señalan como elementos comunes que:

- Aunque no existe una postura homogénea en relación con el fenómeno del CC está incorporado al discurso público.

- La población ha oído hablar del calentamiento global aunque no tiene una postura homogénea al respecto.

- La población considera que se le debe dar más importancia, se percibe según el nivel formativo de la población, según aumenta es mayoritaria la opinión en relación con el CC.

- El nivel de información en relación con el tema es insuficiente, por lo que afecta la percepción del fenómeno y la conducta a seguir en relación con él.

Al respecto resultan interesantes los resultados de los estudios de (Cuevas, 2010: p 25), en relación con la resiliencia el autor aseveró que el éxito de la integración del concepto de resiliencia dentro de los planes de manejo requiere de los siguientes puntos:

1- Que los planes de manejo tengan claros procedimientos para anticipar y responder las amenazas ambientales;

2- Que los planes de manejo deben de considerar la integración del conocimiento biológico de los ecosistemas y el desarrollo de políticas a corto, mediano y largo plazo que permitan el entendimiento de los procesos que operan en una diversidad de escalas espacio-temporales que faciliten el uso de la resiliencia como indicador del estado de conservación, y que a su vez incrementen las posibilidades de identificar, controlar y responder a las amenazas ambientales; 
3- El conocimiento del manejo de los ecosistemas debe ser movilizado a diferentes redes sociales e institucionales que permitan un mejor análisis y ejecución de este y

4- El monitoreo de los cambios en las características de las especies y el vínculo de éstas con factores ambientales pueden aumentar el entendimiento de la resiliencia y el papel de los procesos sociales.

En Cuba la preocupación por el CC ha aumentado en los últimos años, a consecuencia de los diferentes fenómenos meteorológicos que han causado daños severos a la población del país.Como respuesta a su enfrentamiento se aprobó en abril del 2017 por el Consejo de Ministros de Cuba el Plan de Estado para el Enfrentamiento al Cambio Climático (Tarea Vida) y tiene su génesis em el pensamiento del líder Fidel Castro quien consideró la necesidad de salvar la vida em el planeta e insistió reiteradamente en la importancia de la preservación del medio ambiente para las generaciones futuras

Con la aprobación del Plan de Estado para el enfrentamineto al Cambio Climático, se ratificó una vez más la posición de Cuba como país de avanzada a nivel mundial en la lucha contra lo que es considerado el más graves desafío ambiental a encarar por la humanidad en la presente centuria.

El mismo está sustentado en un rico caudal de resultados científicos y tecnológicos acumulado durante más de 20 años de investigaciones durante las cuales se pudieron identificar los impactos actuales y futuros de dicho proceso sobre el archipiélago cubano. Se trata de un programa conformado por cinco acciones estratégicas y 11 tareas, dirigido básicamente a contrarrestar los posibles daños en las zonas vulnerables, preservando ante todo la vida de las personas.

El Plan de Estado para el Enfrentamiento al Cambio Climático tuvo entre sus antecedentes em el estudio denominado Impacto del cambio climático y medidas de adaptación en Cuba, desarrollado en la década de los 90 del siglo xx y donde intervinieron numerosos especialistas de diferentes centros científicos. Ellos coincidieron en que las actividades humanas que generan la contaminación ambiental y el cambio climático global afectan los niveles de resiliencia actuales y futuros y por lo tanto la estabilidad de los ecosistemas con incidencia en la sociedad. 
Otro importante aporte al respecto está contenido en los resultados del Macroproyecto sobre Peligros y Vulnerabilidad de la zona costera cubana para los años 2050 y 2100 , el cual es enriquecido de forma permanente por medio de un monitoreo constante de los especialistas.

La Tarea Vida, tiene un alcance y jerarquía superiores a todos los documentos aprobado anteriormente en relación con el tema, y su instrumentación demandará a la ciencia un programa de acciones progresivas a corto (2020), mediano (2030), largo (2050) y muy largo plazo (2100).

Representa, sin duda, la expresión práctica más abarcadora del compromiso del Gobierno cubano de trabajar desde ahora por la protección de las actuales y futuras generaciones de cubanos frente a las consecuencias del cambio climático.

\section{CONCEPCIÓN TEÓRICO METODOLÓGICA DE LA EDUCACIÓN AMBIENTAL COMUNITARIA NO ESCOLARIZADA (EAC) PARA EL ENFRENTAMIENTO Y ADAPTACIÓN AL CAMBIO CLIMÁTICO: FUNDAMENTOS QUE LA SUSTENTAN.}

La concepción de educación ambiental comunitária no escolarizada defendida por (Castro, 2016:p 21), Idea rectora del presente articulo tiene como elemento que la distingue: el carácter desarrollador de la personalidad, el cual garantizará en el sujeto la apropiación activa y creadora de la cultura ambiental a partir de su propia experiencia; a la vez, propiciará el auto-perfeccionamiento constante del sujeto, de su autonomía y autodeterminación en íntima conexión con los procesos de socialización sustentados en lo comunitario como cualidad,

Lo comunitário como cualidad es defendido por (Alonso, 2009: p. 151). Para esse autor la: "[comunidad] es un grupo social donde transcurren procesos de cooperación y participación en torno a un proyecto colectivo [...]"

En la concepción de comunidad asumida por esse autor se potencian epistemes que fortalecen el enfoque social de la comunidad, lo cual en este artículo no niega reconocer las particularidades del espacio. Esa integración de lo social, con el resto de las particularidades del contexto es una fortaleza para la concepción totalizadora y sistémica del medio ambiente y hace posible el diagnóstico integral de la comunidad. 
Desde la idea de Alonso (2009) "[...] el desarrollo comunitario es despliegue de lo comunitario en el grupo social como expansión del vínculo de simetría social a su interior, a través de procesos de cooperación, participación, y proyecto comunitario [...]". (p. 151)

A partir de esa premisa, cualquier acción que se desarrolle en una comunidad deberá no solo ajustarse a las características del contexto, sino potenciar también los indicadores de lo comunitario por diferentes vías. Esa idea es una fortaleza para la educación ambiental comunitária no escolarizada, la cual se concretará en un proyecto colectivo contextualizado y desarrollará um sujeto activo y protagónico.

Teniendo en cuenta que la sociedad actual demanda de ese sujeto activo que transforme y cambie su comportamiento, en relación con el resto de los compoentes del medio ambeinte, en el presente trabajo se asume que la educación ambiental comunitaria, está sostenido en tres ideas fundamentales:

Primero: la educación es todo proceso de influencia, de configuración o de desarrollo del hombre, al mismo tiempo que el efecto de esa influencia, de esa configuración o de ese desarrollo, (Chávez, 2005: p 21).

Segundo: el medio ambiente como sistema multidimensional de complejas interacciones en continuos estados de cambio, con dimensiones espacio temporal lo que significa interpretarlo en la categoría de sistema ambiental, caracterizado según (González, 2006: p p. 197) por cuatro atributos principales:

1-Composición: Elementos abióticos, bióticos y socioeconómicos.

2-Estructura: Diferenciada vertical y horizontalmente.

3-Funcionamiento: Procesos de entrada, circulación y salidas del sistema.

4-Jerarquía: Define los diferentes rangos taxonómicos sobre la base de los cuales se establecen niveles de subordinación e interrelaciones.

Tercero: la comunidad como grupo social donde se gestan procesos de participación, cooperación y conciencia crítica alrededor de un proyecto común, (Alonso, 2009: p 26).

Desde esos presupuestos se argumentan a continuación los fundamentos que sostienen la concepción de educación ambiental comunitária no escolarizada. Los que fueron enriquecidos en la práctica durante los procesos de intervención. 
Esa concepción tiene como elemento que la distingue: su carácter desarrollador, el cual garantizará en el sujeto la apropiación activa y creadora de la cultura a partir de su propia experiencia; a la vez, propiciará el autoperfeccionamiento constante del sujeto, de su autonomía y autodeterminación en íntima conexión con los procesos de socialización.

La responsabilidad y compromiso social son valores que se desde un papel activo del sujeto implicado, consciente y transformador al asimilar su realidad de forma dinámica y real, lo cual favorece la formación de valores que conducen a transformaciones en los comportamientos para el uso sostenible del MA.

La función desarrolladora es outra de lãs bondades de la prouesta, se concretiza, en la construcción de proyectos comunes que asumen los sujetos. Desde esa óptica, la intencionalidad y finalidad del proceso trasciende las propuestas tradicionales para emerger como transmisión de cultura, como transformación de sí mismo y como transformación de la realidad ajustada al contexto.

Al potenciar la función desarrolladora en la educación ambiental comunitaria no escolarizada, se contribuye también a que "[...] la comunidad se ocupe de sus propios problemas y se organice para resolverlos ella misma, desarrollando sus propios recursos y potencialidades y utilizando los ajenos [...]" (Fals, 1991).

Es importante que dicho proceso tribute a que los sujetos se auto desarrollen como tal, sean activos y protagónicos asumiendo la condición de sujetos de dicha práctica; es decir, se necesita promover procesos de formación de ellos desde "[...] una participación desalienante, conscientizante y socializadora como modo de contrarrestar los efectos ideológicos enajenantes de estructuras dependientes [...]". (Montero, 1989, p. 36)

Todo lo anterior permite el logro de la intencionalidad emancipatoria y auto desarrolladora del proceso, lo cual se consigue al expresar en la capacidad que se alcanza para promover la autogestión, el autodesarrollo de los sujetos, potenciando la acción de transformación y su rol como sujeto transformador de la realidad en función de sus propias necesidades y recursos.

Al mismo tiempo, el centro de poder lo asume el grupo con el protagonismo de las acciones por los propios sujetos, actores, gestores y facilitadores que 
pertenecen al campo de acción.

A partir de estas reflexiones y de los fundamentos antecedentes, aportados por las ciencias Castro (2016) propone contextualizar la Educación Ambiental Comunitaria (EAC) no escolarizada, como:

\begin{abstract}
Un proceso educativo no escolarizado que constituye una dimensión de la educación integral de todos los ciudadanos, orientada a la apropiación de conocimientos, hábitos, habilidades, actitudes, valores y comportamientos, que potencien la armonización de las relaciones entre los seres humanos y de ellos con la sociedad y la naturaleza para propiciar la orientación y conducción de los procesos económicos, sociales y culturales hacia el desarrollo sostenible. Este proceso es eminentemente humanista, emancipador, desarrollador y potenciador de la conciencia crítica de los sujetos comunitarios a través de la potenciación de procesos de participación, y cooperación, que se concretaran en proyectos de autodesarrollo comunitario. (Castro, 2016, p. 26)
\end{abstract}

Esos elementos expresan las cualidades esenciales de la educación ambiental comunitaria no escolarizada, cuyo desarrollo se alcanza en el sujeto individual y colectivo toda vez que se fortalece su protagonismo. Son elementos que tipifican el proceso:

- Transcurre fuera del contexto escolar y aglutina a toda la sociedad, las organizaciones e instituciones.

- Surge del interés de la sociedad y no es necesario sea organizado por la escuela.

- Fortalece la participación y preparación de los líderes, gestores comunitarios, facilitadores y pobladores en general, de forma que se conviertan en agentes socializadores de los saberes adquiridos emn la experiência.

- Concibe el proceso de EAC con un carácter dialéctico, en el que el sujeto desempeña un rol activo, participando y por tanto otorgándosele poder, protagonismo, implicación, responsabilidad, compromiso y facilitando las interrelaciones entre los diferentes agentes socializadores.

- Reconoce la complejidad del proceso, por lo que precisa que un profesional preparado acompañe y facilite al grupo los aprendizajes durante la intervención hasta tanto este haya aprendido las herramientas para continuar solo.

- El proceso educativo se conduce tomando como base la identificación de las contradicciones como fuente del desarrollo, lo que significa descubrir las 
potencialidades y mostrar habilidad para la solución de los problemas y concretar las acciones en el desarrollo del proyecto.

- La concepción del proceso educativo es multilateral, porque intervienen múltiples factores, agentes y agencias socializadoras que influyen en las esferas cognitivas, afectiva y volitiva de los sujetos que participan.

- La conducción metodológica del proceso, hace posible su sostenibilidad, ya que se proyecta hacia el futuro, expresado en un proyecto común concebido a corto, mediano y largo plazo.

Al asentarse en el paradigma sociológico del autodesarrollo, se asume por convicción la formación y el desarrollo de valores que permitan el comportamiento protagónico y responsable de la sociedad em relación con el enfrentamiento al CC

Asumiendo en su concepción:

- La formación de un hombre integral, libre; un hombre que piense, sienta, valore, crea, actúe.

- Un sujeto humanista, participativo, cooperativo y crítico de sí mismo y de su realidad, capaz de transformar la problemática a partir de actitudes responsables.

- Enfoca los presupuestos epistemológicos y axiológicos de la actividad humana con un sentido crítico.

- Fortalece en las personas la capacidad de ser sujetos activos y protagonistas del proceso; es decir, de conformar conscientemente las acciones educativas.

- Defiende la educación como práctica social y demuestra que no es estática, ni rígido sino desarrolladora y cambiante.

- Promueve una interpretación dialéctica del sujeto en el proceso educativo.

- Orienta las acciones a partir de la participación colectiva, dentro de un paradigma emancipador.

- Brinda una concepción de la vida y el ideal de hombre que se quiere alcanzar, y orienta la finalidad educativa.

- Contribuye a consolidar el carácter socializador de la educación, fuera de los marcos de la escuela.

- Vigoriza la idea de vínculos simétricos entre los miembros del grupo así como, con otros grupos y sujetos del exterior. 
- Incide de manera permanente en el proceso educativo por medio de los epistemes que lo identifican.

- Se orienta hacia el futuro por medio de proyectos colectivos, traspasa la escuela para involucrar otros agentes y agencias socializadoras.

- Además, vigoriza el papel de la educación en la conformación del ser social desde una forma novedosa y facilita la aprehensión de la realidad como premisa para el cambio.

- En la labor educativa el proceso de apropiación de la cultura transcurre a través de la actividad como proceso que mediatiza la relación entre el hombre y su realidad objetiva. A través de ella, el hombre modifica la realidad, se forma y se transforma a sí mismo.

La educación ambiental comunitaria no escolarizada, en este caso, no está dirigida solo al espacio físico de un territorio sino que enriquece esa concepción al potenciar al sujeto como protagonista de la acción para el enfrentamiento al CC.en cada território. Desde los fundamentos psicológicos se sustenta en el enfoque histórico-cultural de Vigotsky, en particular en lo referido a que:

- La subjetividad es condición para la socialidad y resultado de la participación en la actividad social práctica.

- La subjetividad reproduce y produce las relaciones sociales, por lo tanto, su desarrollo exige participación en la actividad social, en tanto esta modela su forma, contenido y niveles de desarrollo.

- La concepción de las mediaciones, como expresión de la noción de síntesis dialéctica y procesualidad, subyace a toda la obra vygotskyana.

- El posicionamiento emancipador, desde el énfasis en las posibilidades del sujeto de analizar la relación armónica con la naturaleza y la sociedad.

- Contribuye al establecimiento de una adecuada comunicación basada en el respeto y reconocimiento del otro.

- Ofrece la posibilidad de que las personas se transformen en sujetos de la acción social, en la medida que la actividad colectiva sea expresión de su propia voluntad.

- Facilita que el hombre sea sujeto de una relación de recíproca determinación y transformación, con una realidad que lo trasciende y a la que a su vez modifica. 
Desde el punto de vista pedagógico, se sustenta en las leyes, principios y categorías de las Ciencias Pedagógicas, en particular al abordar la modalidad educativa de la educación no escolarizada. Asume los enfoques de la Pedagogía Social como disciplina que atiende lo teórico y lo práctico como dos dimensiones de una misma realidad, su campo de intervención es el espacio socio-comunitario de trasmisión de valores ciudadanos con la finalidad de superar las contradicciones existentes en los diferentes grupos sociales, escenarios comunitarios.

Se organiza a partir de situar al facilitador como centro de toda la acción educativa y se le convierte en sujeto activo de su conocimiento.Desde el punto de vista didáctico se sustenta en la Didáctica Crítica, como ciencia teórico-práctica que orienta la acción reconstructora del conocimiento, en un contexto de enseñanza-aprendizaje, mediante procesos tendencialmente simétricos de comunicación social, tomando al grupo como escenario fundamental de este proceso y desde el horizonte de una racionalidad emancipadora.

La Didáctica Crítica, es mdular en la concepoción de EAC no escolarizada ya que desde su enfoque emancipador, trata de cambiar a los sujetos como personas individuales y sociales, sus actitudes, sus valores, sus juicios, sus sentimientos, sus capacidades y actitudes.

En la medida en que se cambia al sujeto en lo individual a través de lo grupal se cambia su proyección como sujeto transformador de la sociedade. Influiras en el sujeto de un modo emancipador; por eso, bajo esta concepción didáctica, enseñar es transformar.

La metodología de la Didáctica Crítica, es una práctica participativa, crítica, que favorece el debate, la reflexión, y la discusión en pequeños grupos. Utiliza como métodos novedosos, dinámicas vivenciales, mesas redondas, paneles de discusión, estudios de caso, simulación de roles y juegos dramáticos, entre otros. Desde esta concepción, la visión del mundo que trae cada participante desde su experiencia cultural, educativa, social, política, y desde las múltiples mediaciones generacionales, de género, étnica y social, enriquece el debate sobre las distintas problemáticas sociales y el papel de los sujetos como ciudadanos protagonistas de su autodesarrollo.

En lo sociocultural incluye el conocimiento de valores, actitudes y 
expectativas que se desean transmitir por médio de la (EAC) no escolarizada. La misma permite valorar el aporte que la cultura cotidiana puede ofrecer para enriquecer el desarrollo de la cultura ambiental en la población; que no se limita a la escuela, un grupo o una clase, sino que también incluye a toda la sociedad, sus valores y actitudes y que responden a un contexto determinado.

El sustentar los fundamentos teóricos de partida, desde los referentes anteriormente expresados, es lo que permite aseverar que el proyecto común que se construya por los sujetos es emancipador, y desarrollador, por cuanto garantiza en el individuo la apropiación activa y creadora de la cultura, propiciando así el desarrollo de su autoperfeccionamiento constante, de su autonomía y autodeterminación, en íntima conexión con los necesarios procesos de socialización, compromiso y responsabilidad social.

Entre los principales resultados alcanzados por los autores en la proyección de la concepción de la educación ambeintal comunitária no escolarizada, se aprecia la transformacion de la sociedad.

. Para ello es necesario:

- Desarrollar, investigar, divulgar, preservar y difundir la problemática ambeintal

- Crear mecanismos para promover la participación de las comunidades en la toma de decisiones, la planificación, el desarrollo y los beneficios derivados de los proyectos de EAC.

- Diseñar proyectos con la cooperación de las instituciones para promover la preservación, conservación, aprovechamiento y manejo sustentable de los espacios naturales y socio culturales,

- Contribuir a la capacitación, y concientización de la población en relación con la problemática ambeintal

- Posibilitar que los sujetos transformen su problemática a partir de su incidencia en proyectos de educación ambiental comunitaria que tributen al desarrollo de la cultura ambiental, como premisa para fortalecer el desarrollo sostenible.

- Ofrecer a la población el conocimiento necesario para la interpretación ambiental del patrimonio natural y cultural, a la vez que la propia población local se comporta de forma responsable. 
- Proponer un código de comportamiento, a fin de enfatizar en la actitud, comportamientos y valores que deberán mostrar los sujetos en relación con el uso sostenible del medio ambiente. 


\section{CONSIDERACIONES FINALES}

Los resultados del presente estudio muestran que el cuestionamiento de la crisis ambiental a nivel planetaria y el impacto de problemas como el cambio climático em los diferentes espacios, tiene su repercusión em la educación de cada país. Cuba desarrolla importantes investigaciones em esa dirección, de ellas emergen reflexiones, estratégias y proyectos que exponen los resultados alcanzados

Como tendência de lãs investigaciones cubanas está el reconocimiento del caracter totalizador del médio ambiente superando el enfoque naturalista, asi como potenciar la EA desarrolladora en el contexto escolar y su proyección desde la escuela en los asentamientos.

Los resultados aportados por los autores del presente artículo va más allá de la Via No Formal organizada por las instituciones escolares. A partir de lãs expriencias adquiridas en la práctica investigativa de los autores y sitematizadas desde el 2010 hasta la fecha se percibe que la EAC no escoalrizada tiene potencialidades para ser aplicada en el enfrentamiento y adaptación al cambio climático segun particularidades de cada contexto.

Su valor está contenido em los siguientes argumentos:

La propuesta de una educación ambiental comunitária no escolarizada para el enfrentamiento y adaptación al CC se adquiere y construye; la misma se expresa en un sistema de conocimientos, hábitos, habilidades, actitudes y valores, a partir de su inserción en los procesos que se suceden en los contextos afectados por el Cambio Climático, de manera directa o indirecta.

Está mediada por la cosmovisión de los sujetos comunitarios en relación con ese fenómeno y alcanza un valor teórico y metodológico en los estudios del medio ambiente en espacios vulnerables La educación ambiental comunitária, no escolarizada constituye por tanto la vía efectiva para la adaptación y enfrentamiento al CC.

Se potencia la armonización de las relaciones entre los seres humanos y de ellos con la sociedad y la naturaleza para propiciar la orientación y conducción de los procesos económicos, sociales y culturales hacia el enfrentamiento al cambio climático em pos del desarrollo sostenible.

El proceso de EAC es eminentemente humanista, emancipador, 
desarrollador y potenciador de la conciencia crítica de los sujetos comunitarios a través de la potenciación de procesos de participación, y cooperación, que se concretarán en proyectos de autodesarrollo comunitario para el efrentamiento al cambio climático

Sobre la base de los resultados obtenidos en la sistematización de los fundamentos teórico-metodológicos que sustentan el sistema construido, los resultados concretos obtenidos del diagnóstico aplicado sobre la realidad cubana en general y de los sujetos muestreados en particular, y finalmente, por la evaluación y sistematización de la planeación e instrumentación de lãs investigaciones realizadas se aprecia también que es una concepción integrada que se construye; la misma se expresa en un sistema de conocimientos, hábitos, habilidades, actitudes y valores, a partir de su inserción en los procesos.

Está mediada por la cosmovisión de los sujetos comunitarios hacia la sostenibilidad de medio ambiente, y alcanza un valor teórico y metodológico inseparables en los procesos de transformación comunitaria

La educación ambiental comunitaria no escolarizada constituye una vía efectiva para la formación de la cultura ambiental si se concibe esta como un proceso educativo no escolarizado que constituye una dimensión de la educación integral de todos los ciudadanos, Por médio de la apropiación de conocimientos, hábitos, habilidades, actitudes y valores, fortalece la armonia de las relaciones entre los seres humanos y de ellos con la sociedad y la naturaleza para propiciar la orientación y conducción de los procesos económicos, sociales y culturales hacia el desarrollo sostenible.

Ese proceso es eminentemente humanista, emancipador, desarrollador y potenciador de la conciencia crítica de los sujetos comunitarios a través de la potenciación de procesos de participación, y cooperación, que se concretarán en proyectos de autodesarrollo comunitario. Esos proyectos se construyen de forma participativa por los sujetos, para lo cual se utilizan métodos y técnicas grupales como entrevista en profundiad, grupos focales y de reflexión.

El proceso se despliega con carácter emancipador, participativo y desarrollador a través de las sesiones de trabajo que se organizan para la intervención. Para el desarrollo de lãs sesiones de trabajo se utilizan diferentes vias como: la capacitación de los facilitadores, interpretación ambiental, talleres 
reflexivos y la planeación e instrumentación del sistema de acciones que tributan a conformar el Proyecto de educación ambiental comunitaria na escolarizada dirigida al desarrollo de la cultura ambiental para el enfrentamiento al cambio climático.

El sentido de la concepción propuesta aquí, es entregarle a lãs futuras generaciones herramientas teórico-metodológicas que pueden hacer sostenible el medio ambiente donde la sociead defienda com responsabilidad su belleza, riqueza en su diversidad biológica, y sostenibilidad de la vida, para que em el futuro "La Primavera no sea silenciosa". Esa es la aspiración que encierra este artículo, fin por excelência humanista nacido del respeto a la vida y del amor hacia la especie humana. 


\section{REFERENCIAS}

ALONSO, Joaquín. La comunidad y el vínculo comunitario". El Desarrollo Local Comunitario desafíos actuales para América Latina.Universidad Central "Marta Abreu" de Las Villas, Cuba: Edit Feijóo.2009.

BAIGORRI A. Cambio global, vulnerabilidad y resiliencia: El papel de las Ciencias Sociales en Transiciones ambientales y participación. Edit Amarú. España. 2012.

CASTRO Georgina. Proyecto de educación ambiental comunitaria para el desarrollo de la cultura ambiental turística en la población del Batey Reforma. Tesis en opción al grado científico de Doctor en Ciencias de la Educación. Cuba. 2016.

CUEVAS Pablo. Laboratorio de Ecología de Interacciones Bióticas, Facultad de Biología, Revista Biológicas | Vol. 12, No. 1 | Julio 2010.

CHÁVEZ, J.\& col Acercamiento necesario a la Pedagogía como ciencia. Ciudad de La Habana: Edit. Pueblo y Educación. 2005.

FALS B, O. Acción y conocimiento. Cómo romper el monopolio con investigación-acción participativa. Santa Fe de Bogotá: Edit. Cinep. 1991.

GONZALES Manuel Gestión ambiental de los impactos del turismo. Edit. ABIAYALA. Quito. Ecuador, 2006.

MATEO, José Manuel La dimensión espacial del desarrollo sostenible: una visión desde América Latina: Edit UH, 2012.

MONTERO Maritza. La participación: significado, alcance y límites.1989. Consultado 15 de marzo 2015.2 Disponible en: http://www.ipap.sg.gba.gov.ar/doc/mmar.doc, acesso 02/11/2019.

ROQUE Martha.Estrategia para la formación de la cultura ambiental para profesionales cubanos de nivel superior orientada al desarrollo sostenible Tesis en opción al grado de Doctor en Ciencias de la Educación, inédita. La Habana. Cuba. 2003.

SANTOS MILTON. Espaço e método.Sao Paulo, Nobel, 1992. 